\title{
Fluorescein angiography in toxaemia of pregnancy
}

\author{
WILLIAM C. MABIE ${ }^{1}$ AND RICHARD R. OBER ${ }^{2}$ \\ From the Departments of ${ }^{1}$ Obstetrics and Gynecology and ${ }^{2}$ Ophthalmology, \\ University of Southern California School of Medicine, Los Angeles, \\ California 90033, USA
}

SUMmARY The clinical and fluorescein angiographic findings in 2 cases of retinal detachment occurring in toxaemia of pregnancy are presented. Both cases showed angiographic evidence of abnormal choroidal leakage of dye unassociated with signs of retinal angiospasm. Both cases also showed angiographic evidence of delayed filling of the choriocapillaris. We believe this finding has not previously been reported. These observations provide further clinical evidence that retinal detachment in toxaemia of pregnancy is secondary to choroidal damage.

Toxaemia of pregnancy is characterised by generalised oedema, proteinuria, and hypertension, and generally occurs in the third trimester of pregnancy. The pre-eclamptic state progresses to eclampsia if convulsions supervene. Spontaneous retinal detachment in toxaemia was first reported by Von Graefe in $1855 .{ }^{1}$ Although retinal detachment is now a well recognised complication of toxaemia of pregnancy, it is very uncommon. ${ }^{2-6}$

The pathogenesis of retinal detachment in toxaemia of pregnancy is not fully understood, and both the retina and the choroid have been implicated as the source of the subretinal fluid in this condition. ${ }^{7-12}$ Previous reports in the literature describing fluorescein angiographic findings have suggested that detachment is secondary either to choroidal vascular damage alone $\mathrm{e}^{13}$ or possibly to both retinal and choroidal vascular damage. ${ }^{14}$

This report describes the clinical findings in 2 cases of retinal detachment occurring in toxaemia of pregnancy. Fluorescein angiography showed evidence of choroidal leakage of dye as well as delayed filling of the choriocapillaris. We believe the latter finding has not been reported in the literature. These observations lend support to the hypothesis that retinal detachment in toxaemia of pregnancy is secondary to choroidal vascular damage alone.

\section{Case reports}

CASE 1

A 20-year-old primigravid, Hispanic woman was admitted to hospital in labour with a placental

Correspondence to Richard R. Ober, MD, Estelle Doheny Eye Foundation, 1355 San Pablo Street, Los Angeles, California 90033, USA. abruption at approximately 34 weeks gestation on 21 September 1976 with a 1-day history of decreased vision in the right eye. The past medical history was unremarkable.

Physical examination showed a blood pressure of $150 / 100 \mathrm{mmHg}, 1+$ oedema, and mild deep tendon hyperreflexia. No fetal heart tones were heard, and fetal death was confirmed by fetal monitor. Ocular examination showed a visual acuity of $6 / 60(20 / 200)$ in the right eye and $6 / 6(20 / 20)$ in the left eye. External examination was normal except for a $1+$ afferent pupillary defect on the right. Biomicroscopic examination of both eyes was normal. Ophthalmoscopic examination showed a serous retinal detachment involving the posterior pole of

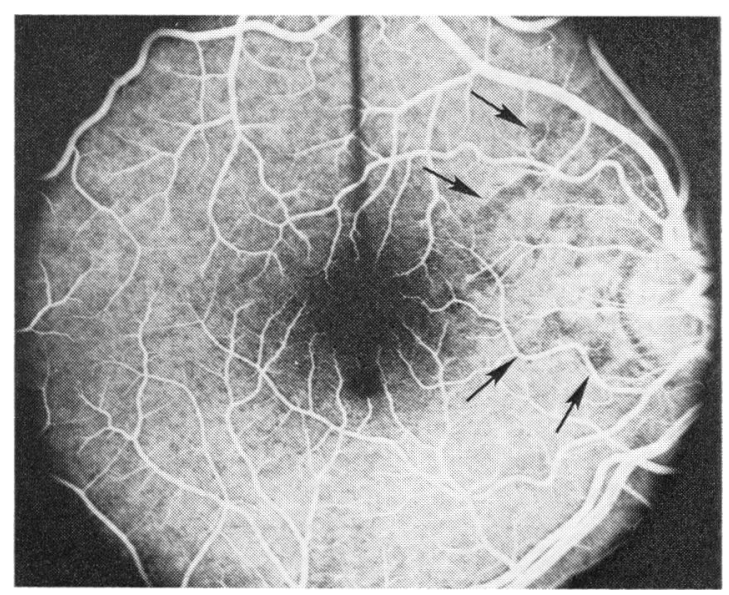

Fig. 1 Case 1. Fluorescein angiogram in early venous phase of right eye. seven days after delivery, showing delayed filling of choriocapillaris surrounding optic disc (arrows). Note normal retinal vasculature. 


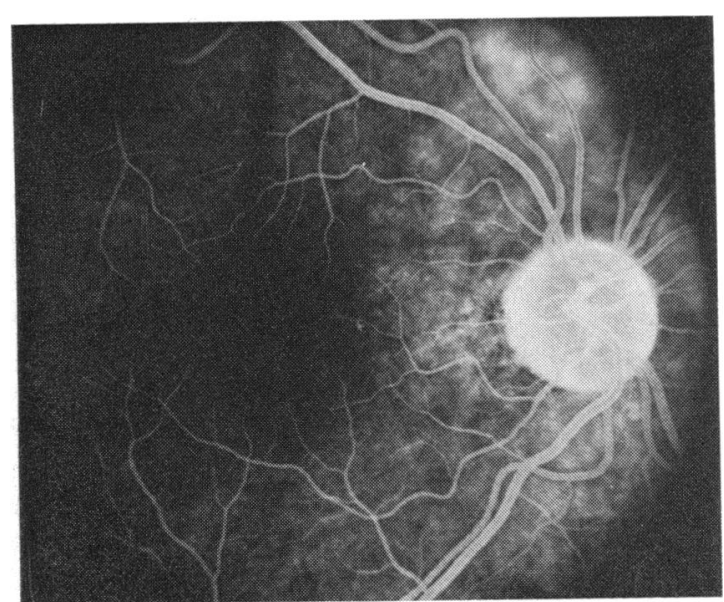

Fig. 2 Case 1. Fluorescein angiogram of right eye in late venous phase showing choroidal dye leakage surrounding optic disc.

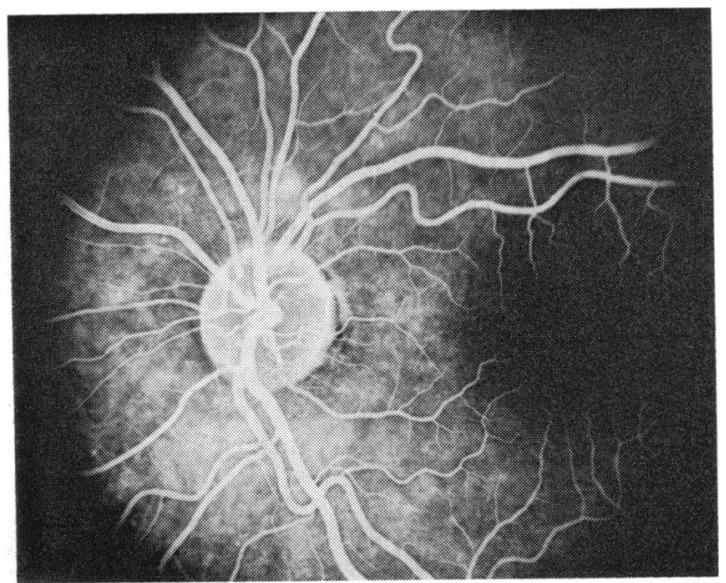

Fig. 3 Case 1. Fluorescein angiogram of left eye in late venous phase showing choroidal dye leakage surrounding optic disc.

the right eye. A peripapillary serous retinal detachment was present on the left without macular involvement. No retinal cotton-wool spots, haemorrhages, or arteriolar constriction were noted.

Urine analysis showed $1+$ proteinuria. Other laboratory studies, including complete blood count, platelet count, fibrinogen levels, prothrombin time, and renal and liver function tests, were normal.

After a stillborn infant was delivered the vision in the right eye began to improve and the serous detachments gave signs of resolution. One week post partum fundus examination showed mild pigmentary disturbances in the right macula. A 1.5 disc diameter area of neurosensory retina was raised inferotemporal to the right macula. Fluorescein angiography showed delayed filling of the choriocapillaris surrounding the optic disc in the right eye (Fig. 1). In the late venous phase there were numerous areas of dye leakage surrounding the optic disc bilaterally at the level of the choriocapillaris and pigment epithelium. These areas of hyperfluorescence enlarged and gradually coalesced (Figs. 2 and 3). There was mild staining of the subretinal fluid inferotemporal to the right fovea. No abnormalities of the retinal vasculature were noted.

The patient was last examined on 1 November 1976 , and vision was $6 / 6(20 / 20)$ in both eyes. The fundus examination was normal except for some minimal pigmentary changes in the right macula. Antihypertensive medications which were required during the patient's time in hospital were discontinued 12 days post partum, and the patient remained normotensive during the follow-up period.

\section{CASE 2}

A 36-year-old gravida-3, para-2, Hispanic woman was admitted to hospital at approximately 32 weeks gestation on 22 December 1975, with a 1month history of progressive generalised oedema and shortness of breath and a 1-week history of bilateral decreased vision. She had had a fever and a swollen left leg for 2 days before admission.

Past medical history revealed that she had been treated intermittently for hypertension for several years and had 2 previous caesarean sections for unknown reasons, producing normal infants.

Physical examination showed an obese $(270 \mathrm{lb}$ $(122 \mathrm{~kg})$ ) obtunded patient with anasarca. The blood pressure was $200 / 120 \mathrm{mmHg}$, pulse rate 140 , temperature $102^{\circ} \mathrm{F}\left(38.9^{\circ} \mathrm{C}\right)$, and respiratory rate 40 . Cellulitis of the lower left leg was present Deep tendon reflexes showed unsustained clonus. On ocular examination she had a visual acuity of hand motion in each eye. External and biomicroscopic examination of each eye was normal. Ophthalmoscopic examination disclosed bilateral bullous serous retinal detachments. No retinal cotton-wool spots, haemorrhages, or vascular abnormalities were noted. Several small, whitish subretinal lesions were seen in the posterior pole of both eyes.

Abnormal laboratory studies were consistent with microangiopathic haemolytic anaemia ${ }^{15}$ and included: haemoglobin $8.6 \mathrm{~g} / \mathrm{dl}$, haematocrit $32 \%$, white blood count $19 \times 10^{\circ} / 1$, platelet count $40 \times 10^{\%} / 1$, and corrected reticulocyte count $4 \%$ A peripheral smear showed a moderate number of fragmented red cells. Abnormal clotting function tests included a weakly positive protamine sulphate test and a. slightly prolonged bleeding time. Fibrinogen level, 
prothrombin time and partial thromboplastin time were normal. Bone marrow examination showed a hypercellular marrow with increased megakaryocytes. Urine analysis registered a $4+$ proteinuria, $1+$ biliburin, and urobilinogen 8 Ehrlich units (normal up to 1). Abnormal or borderline low blood chemistries included: total biliburin 2.1 $\mathrm{mg} / \mathrm{dl} \quad(35.9 \mu \mathrm{mol} / \mathrm{l})$ (normal 0.2-1.5 (3.4-25.6)), SGOT 170 Karmen units (normal 5-40), alkaline phosphatase 4.4 Bessey-Lowry units (normal 1-3), blood urea nitrogen $25 \mathrm{mg} / \mathrm{dl}(8.9 \mathrm{mmol} / \mathrm{l})$ (normal 6-25 $(2 \cdot 1-8 \cdot 9))$, creatinine $1.7 \mathrm{mg} / \mathrm{dl}(150 \mu \mathrm{mol} / \mathrm{l})$ (normal 0.4-1.3 (35-115)), sodium $127 \mathrm{mEq}(\mathrm{mmol})$ (normal 134-142), potassium $3.7 \mathrm{mEq}(\mathrm{mmol})$ (normal $3 \cdot 7-5 \cdot 1$ ), bicarbonate $18 \mathrm{mEq}(\mathrm{mmol})$ (normal 21-29, chloride $96 \mathrm{mEq}(\mathrm{mmol})$ (normal 96-105), uric acid $9 \cdot 1 \mathrm{mg} / \mathrm{dl}(0.54 \mathrm{mmol} / \mathrm{l})$ (normal $2 \cdot 5-6 \cdot 8(0 \cdot 15-0 \cdot 4))$, glucose $160 \mathrm{mg} / \mathrm{dl}(8.9 \mathrm{mmol} / \mathrm{l})$ (normal 62-110 (3.4-6.1)), total protein $5.5 \mathrm{~g} / \mathrm{dl}$ $(55 \mathrm{~g} / \mathrm{l})$ (normal 6.5-8.3 (65-83)) and albumin $2.6 \mathrm{~g} / \mathrm{dl}(26 \mathrm{~g} / \mathrm{l})$ (normal 3.9-5.0 (39-50)).

The patient was treated with intravenous magnesium sulphate, hydrallazine, penicillin, and gentamicin. Ten platelet packs were required for stabilisation.

Repeat caesarean section was performed, and a slightly macerated stillborn male infant was delivered from a fibroid uterus.

Postoperatively she received 4 units of whole blood; magnesium sulphate and antibiotics were continued, and a wound infection was opened and drained. Methyldopa and hydrochlorothiazide were begun on the third postoperative day. The platelet count and renal and liver function studies returned to

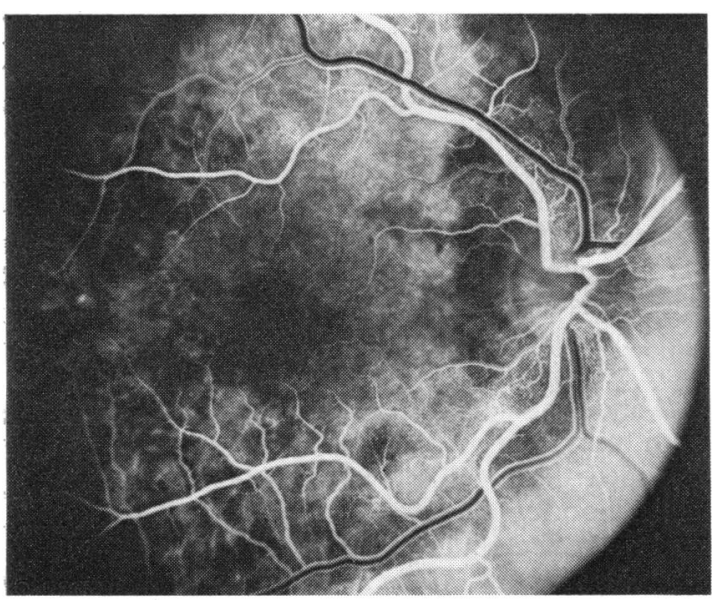

Fig. 4 Case 2. Fluorescein angiogram in arteriovenous phase of right eye, 14 days after delivery, showing delayed filling of choriocapillaris.

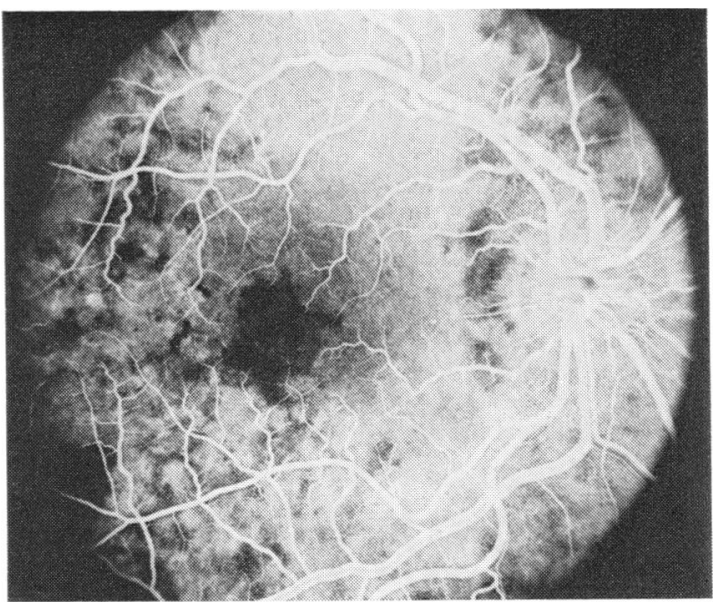

Fig. 5 Case 2. Fluorescein angiogram in early venous phase of right eye confirming delayed filling of choriocapillaris in posterior pole and showing early choroidal leakage. Note normal retinal vasculature.

normal, and the patient lost $60 \mathrm{lb}(27 \mathrm{~kg})$ by diuresis while in the hospital.

On 7 January 1976, 2 weeks post partum, the vision was $6 / 15(20 / 50)$ in both eyes, and the detachments had largely resolved except for inferior subretinal fluid. Both maculae appeared to be slightly oedematous. Diffuse mild subretinal pigmentary disturbance was present in the posterior pole of both eyes. A few scattered whitish lesions were seen in the right eye underlying the macula. Fluorescein angiography of the retinal vasculature appeared normal. In the arteriovenous phase

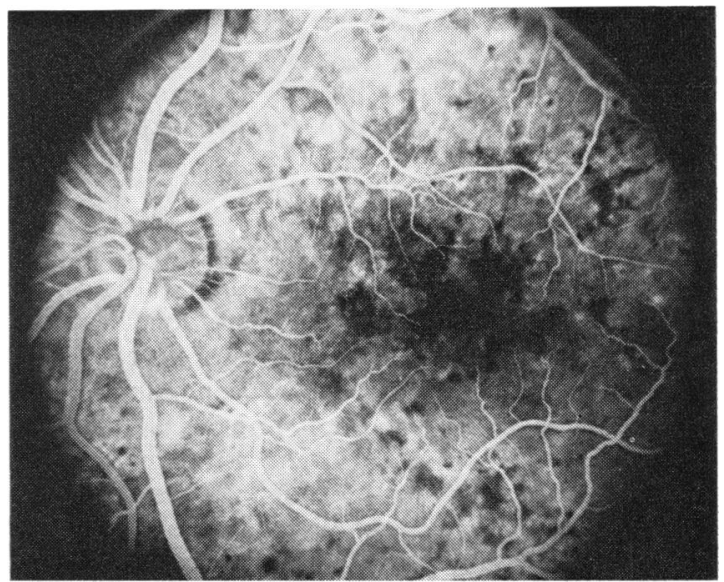

Fig. 6 Case 2. Fluorescein angiogram in late venous phase of left eye showing delaying filling of choriocapillaris and leakage of dye from choroidal vasculature. 
delayed filling of the choriocapillaris persisted into the late phase of the angiogram (Fig. 4-6). Small dots of hyperfluorescence in both eyes were located at the level of the choriocapillaris and retinal pigment epithelium, and this staining increased in the late phase. Mild bilateral peripapillary staining was also present in the late phase of the angiogram.

The patient was discharged on 9 January 1976. She was last seen on 30 January 1976, at which time her vision was 6/12 (20/40) in the right eye and 6/9 $(20 / 30)$ in the left. The fundi were normal except for some mild bilateral pigmentary disturbance in the macula. Her blood pressure remained poorly controlled on antihypertensive medication.

\section{Discussion}

Toxaemia of pregnancy usually occurs in the third trimester in primigravid women, who often have received inadequate prenatal care. Severe arteriolar spasm, evidenced by either segmental or generalised constriction, is the commonest finding in the fundus and has been reported in $70 \%$ of cases of toxaemia. ${ }^{16}$ Retinal haemorrhages, retinal oedema, and cottonwool spots secondary to arteriolar damage may follow. Serous retinal detachments occur in the presence of severe pre-eclampsia or eclampsia, but only rarely. They may be seen in association with severe retinal vasospasm, haemorrhages, and exudates, but have also been reported in the absence of severe retinal arteriolar pathology. ${ }^{17}$

The incidence of serous retinal detachment in toxaemia of pregnancy varies, but all reports have emphasised the rarity of the condition. Fry ${ }^{2}$ noted a $1.2 \%$ incidence of retinal detachment in toxaemia and $10.4 \%$ in eclampsia. Hallum ${ }^{3}$ reported 6 cases in 300 pre-eclamptics. Mittelstrass and Wolf hagen ${ }^{4}$ reported 1 case in 873 eclamptics. Kronenberg ${ }^{5}$ reported 2 cases in 20538 pregnancies, and Bosco $^{6}$ reported 1 case in 18524 pregnancies. We found only the 2 cases in this report in 62832 deliveries at the Los Angeles County-University of Southern California Medical Center during a 5-year period (1974-78) in a patient population with approximately an $8 \%$ incidence of toxaemia. This observation confirms the previously reported low incidence of this condition. We suspect, however, that additional cases might have been discovered if ophthalmological examinations with indirect ophthalmoscopy had been performed on all toxaemic patients.

The pathogenetic mechanism for the production of serous retinal detachment in toxaemia of pregnancy is not fully understood. Clapp ${ }^{18}$ proposed that it was an extension of cerebral oedema. In a report on reversible retinal detachment in renal failure Lapco et al. ${ }^{19}$ felt it was related to generalised oedema. Moore ${ }^{7}$ and Friedenwald 8 believed the retinal detachments were secondary to retinal vascular damage, with Friedenwald presenting pathological evidence of acute necrosis of vessel walls.

In 1911 Verderame ${ }^{12}$ demonstrated choroidal sclerosis in a post-mortem eye from a patient with toxaemic retinal detachment and was the first to postulate choroidal damage as the cause of detachment. Others have subsequently presented experimental, pathological, and clinical evidence that changes in the choriocapillaris and pigment epithelium are responsible. ${ }^{9-11}$ Choroidal ischaemia and spontaneously reversible retinal detachments in 14 of 23 cats were produced by Collier ${ }^{9}$ by injecting a latex microsphere suspension retrograde into the vortex vein. Loss of the external layers of the retina and disruption of the normal pattern of the pigment epithelium were seen histologically. No occlusive effects were noted in the retinal vessels. Collier postulated that the mechanism of the serous detachment involved occlusion of the choriocapillaris, which resulted in increased choroidal hydrostatic pressure producing vasodilation, with subsequent transudation of fluid into the subretinal space. Stern and Ernest ${ }^{10}$ performed microsphere embolic occlusion of the posterior ciliary arteries supplying the choriocapillaris in rhesus monkeys. Small white patches of oedema in the external retina were caused by infarction of the retinal pigment epithelium and external retinal layers and corresponded to focal areas of fluorescein leakage on angiography. These white patches resembled the Elschnig spots seen in malignant hypertension, pre-eclampsia, and collagen vascular disease. Furthermore, retinal detachments developed in 2 monkeys made hypertensive with intravenous noradrenaline during the procedure. The pathological findings in serous retinal detachments in 3 patients with malignant hypertension and 1 with chronic glomerulonephritis were reported by Klein, ${ }^{11}$ who established a direct relationship between ischaemic infarcts of the choriocapillaris and retinal separation. Thrombotic occlusion of the choriocapillaris with fibrinoid necrosis of the walls, the adjacent stroma, and the overlying pigment epithelium was demonstrated in 2 of the 4 necropsied cases. Another pre-eclamptic patient with serous retinal detachment recovered, but was left with small yellowish scars and central pigment deposits (Elschnig spots) thought to represent healed infarcts of the choriocapillaris. Cogan ${ }^{20}$ noted that the ocular lesions of malignant hypertension, as described by Klein, had the same histological appearance as those in disseminated intravascular coagulation (DIC) and suggested systemic 
coagulation as the pathogenetic mechanism. He reported the pathological findings in DIC, which included thrombotic occlusion of the choriocapillaris in the submacular region, choroidal haemorrhage, vacuolisation and disruption of the pigment epithelium with dispersion into the subretinal space, and serous detachment of the retina. Cogan postulated that the vulnerability of the submacular region in systemically induced hypercoagulability is presumably due to haemodynamic peculiarities of the circulation in this region.

Fluorescein angiographic studies of toxaemic patients with serous retinal detachment have been limited, partly because of fear of teratogenic effects on the fetus. ${ }^{21}$ Nevertheless, 2 detailed reports have appeared in the literature. Gitter and associates ${ }^{13}$ provided clinical evidence that the likely pathogenesis of the detachment is localised in the choroid. They reported a severe pre-eclamptic patient with bilateral serous retinal detachment without associated retinal vascular abnormalities. Fluorescein angiography performed prior to delivery showed a normal retinal vasculature, with the exception of a single constricted arteriole, and multiple areas of choroidal dye leakage with pooling of dye beneath the detachments. They suggested that dye leakage was due to transudation of fluid from the choroidal vessels into the subretinal space. Angiography performed 8 days post partum, when visual acuity had returned to normal, showed no leakage or pooling of dye. In contrast, Kenny and Cerasoli ${ }^{14}$ demonstrated clinically that detachment in toxaemia of pregnancy is secondary to changes in both the retinal and choroidal circulation. They performed colour fluorescein angiography in a patient with bilateral toxaemic retinal and choroidal detachments 48 hours post partum which showed abnormal retinal capillary filling as well as abnormalities in the pigment epithelial pattern. Their patient had marked involvement of the retinal vasculature, with narrowed arterioles, cotton-wool spots, and intraretinal haemorrhages.

The patients in the present report provide further evidence that retinal detachment can be secondary to choroidal damage alone. No evidence of retinal vasospasm, retinal haemorrhages, or cotton-wool spots was noted during the initial funduscopic examination in either patient. Fluorescein angiography performed 7 days post partum in case 1 and 14 days post partum in case 2 revealed multiple areas of dye leakage which enlarged with time; they probably represented damaged areas of pigment epithelium which allowed dye to accumulate in the subretinal space. The fluorescein angiogram in case 1 showed delayed filling of the choriocapillaris in the peripapillary region of the right eye and the posterior pole of both eyes in case 2 . The angiographic finding of delayed filling of the choriocapillaris is a feature which has not been described previously in this condition and which occurs in occlusive choroidal vascular disorders such as hypertension, collagen vascular disease, diabetes, and blood dyscrasias. ${ }^{22}$ This differs from the physiologically normal 'patchy choroidal filling', in which the choroid shows early hypofluorescence but fills normally within 2-5 seconds. Presumably the nonperfusion of the choriocapillaris seen in these 2 cases resulted in ischaemia of the overlying pigment epithelium, with consequent late leakage of dye. No evidence of retinal vasospasm was noted in either angiogram. The possibility that retinal arteriolar abnormalities occurred acutely, but later disappeared, cannot be excluded, since the angiograms were done 7 and 14 days post partum.

Bill and Linder ${ }^{23}$ demonstrated leakage of Evans blue dye out of uveal blood vessels of cats after sympathectomy during increase of the systemic blood pressure. In a study of the effects of systolic hypertension on rhesus monkey eyes after ocular sympathectomy Ernest ${ }^{24}$ showed fluorescein leakage of dye from the uveal circulation in these animals that did not occur after ocular sympathectomy or systemic hypertension alone. Potts ${ }^{25}$ has shown an increased blood flow and increased hydrostatic pressure through the macula, the vasculature of which is denser than in the retinal periphery. Although the exact mechanism remains unproved, these hydrodynamic factors, coupled with an alteration of sympathetic neural control in the hypertensive toxaemic patient, may play a role in causing choroidal vascular incompetency with resultant leakage and serous retinal detachment.

We thank Mr Bill Hart and Mr William Ludwick for taking the photographs and Ms Elva Barrera for secretarial assistance.

AdDendum. Since submitting this paper for publication 2 cases of choroidal nonperfusion in toxaemia of pregnancy have been reported. ${ }^{28}$ Owing to predominantly abnormal choroidal vascular patterns the authors implicated choroidal vascular insufficiency as the primary basis for secondary retinal detachments seen in toxaemia of pregnancy.

\section{References}

${ }^{1}$ Von Graefe A. Uber eine Krebsablagerung im Innern des Auges, deren ursprunglicher Sitz zwischen Sclera und Choroidea war. Albrecht von Graefes Arch Klin Ophthalmol $1855 ; 2$ : 214-24.

${ }^{2}$ Fry WE. Extensive bilateral retinal detachment in eclampsia, with complete reattachment: report of two cases. Arch Ophthalmol 1929; 1: 604-14.

${ }^{3}$ Hallum AV. Eye changes in hypertensive toxemia of pregnancy: a study of three-hundred cases. JAMA 1936;
106: 1649-51. 
${ }^{4}$ Mittelstrass H, Wolfhagen O. Die Bedeutung der Augenhintergrundsuntersuchung für die Klinik der eklamptischen Schwangerschaftserkrankungen. Geburtshilfe Frauenheilkd 1948; 8: 671-87.

${ }^{5} \mathrm{~K}$ ronenberg EW. Toxemic retinopathy of pregnancy with special consideration of retinal detachment as complication of pregnancy toxicosis. Ned Tijdschr Geneeskd 1956; 100: 331-8.

${ }^{6}$ Bosco JAS. Spontaneous nontraumatic retinal detachment in pregnancy. Am J Obstet Gynecol 1961; 82: 208-12.

'Moore F. Medical Ophthalmology, 2nd ed. London: Blakiston Company, 1925: 189-90.

${ }^{8}$ Friedenwald JS. Pathogenesis of albuminuric retinitis. Libman Anniv Vols 1932; 2: 453-8.

${ }^{9}$ Collier RH. Experimental embolic ischemia of the choroid. Arch Ophthalmol 1967; 77: 683-92.

${ }^{10}$ Stern WH, Ernest JT. Microsphere occlusion of the choriocapillaris in rhesus monkeys. Am J Ophthalmol 1974; 78: 438-48.

${ }^{11}$ Klein BA. Ischemic infarcts of the choroid (Elschnig spots). Am J Ophthalmol 1968; 66: 1069-74.

${ }^{12}$ Verderame $P$. Uber nichtalbuminurische und albuminurische Netzhautablosung und ihre Wiederanlegung bei Schwangeren. Klin Monatsbl Augenheilkd 1911 ; 49: 452-68.

${ }^{13}$ Gitter KA, Houser BP, Sarin LK, Justice J Jr. Toxemia of pregnancy: an angiographic interpretation of fundus changes. Arch Ophthalmol 1968; 80 : 449-54.

${ }^{14}$ Kenny GS, Cerasoli JR. Color fluorescein angiography in toxemia of pregnancy. Arch Ophthalmol 1972; 87: 383-8.
${ }^{15}$ Fletcher JP. Eclampsia and microangiopathic haemolytic anaemia. Med J Aust 1971; i: 1065-66.

${ }^{16}$ Wagener HP. Arterioles of the retina in toxemia of pregnancy. JAMA 1933; 101: 1380-4.

${ }^{17}$ Wagener HP. Lesions of the optic nerve and retina in pregnancy. JAMA 1934; 103: 1910-3.

${ }^{18}$ Clapp CA. Detachment of the retina in eclampsia and toxemia of pregnancy. Am J Ophthalmol 1919; 2: 473-85.

${ }^{19}$ Lapco L, Weller JM, Greene JA Jr. Spontaneously reversible retinal detachment occurring during renal insufficiency. Ann Intern Med 1965; 63: 760-6.

${ }^{211}$ Cogan DG. Ocular involvement in disseminated intravascular coagulopathy. Arch Ophthalmol 1975; 93: 1-8.

${ }^{21}$ Schatz H, Burton TC, Yannuzzi LA, Rabb MF. Interpretation of Fundus Fluorescein Angiography. St Louis: Mosby, 1978: 37.

${ }^{22}$ Schatz H, Burton TC, Yannuzzi LA, Rabb MF. Interpretation of Fundus Fluorescein Angiography. St Louis: Mosby, 1978: 219-48.

${ }^{23}$ Bill A, Linder J. Sympathetic control of cerebral blood flow in acute arterial hypertension. Acta Physiol Scand 1976; 96: 114-21.

${ }^{24}$ Ernest JT. The effect of systolic hypertension on rhesus monkey eyes after ocular sympathectomy. Am J Ophthalmol 1977; 84: 341-4.

${ }^{25}$ Potts AM. An hypothesis on macular disease. Trans Am Acad Ophthalmol Otoloaryngol 1966; 70: 1058-62.

${ }^{26}$ Fastenberg DM, Fetkenhour CL, Choromokos E, Shoch DE. Choroidal vascular changes in toxaemia of pregnancy. $\mathrm{Am}$ J Ophthalmol 1980; 89: 362-68. 